\title{
Mortality in patients with non-functioning pituitary adenoma is increased: systematic analysis of 546 cases with long follow-up
}

\author{
Georgia Ntali ${ }^{1}$, Cristina Capatina1, Violet Fazal-Sanderson ${ }^{1}$, James V Byrne ${ }^{2}$, \\ Simon Cudlip ${ }^{3}$, Ashley B Grossman ${ }^{1}$, John A H Wass ${ }^{1}$ and Niki Karavitaki,4 \\ ${ }^{1}$ Department of Endocrinology, Oxford Centre for Diabetes, Endocrinology and Metabolism, Churchill Hospital, \\ Oxford, UK, Departments of ${ }^{2}$ Neuroradiology and ${ }^{3}$ Neurosurgery, John Radcliffe Hospital, Oxford, UK and ${ }^{4}$ School \\ of Clinical and Experimental Medicine, Institute of Metabolism and Systems Research, University of Birmingham, \\ Wolfson Drive, Edgbaston, Birmingham B15 2TT, UK
}

\author{
Correspondence \\ should be addressed \\ to N Karavitaki \\ Email \\ n.karavitaki@bham.ac.uk
}

\begin{abstract}
Objective: Non-functioning pituitary adenomas (NFAs) have a prevalence of 7-22/100 000 people. A significant number of patients suffer from morbidities related to the tumor, possible recurrence(s), and treatments utilized. Our aim was to assess mortality of patients with macroNFA and predictive factors.

Design: Retrospective cohort study in a tertiary referral center in the UK.

Methods: A total of 546 patients operated for a macroNFA between 1963 and 2011 were studied. Mortality data were retrieved through the National Health Service Central Register and hospital records and recorded as standardized mortality ratio (SMR). Mortality was estimated for the total and various subgroups with clinical follow-up data.

Results: Median follow-up was 8 years (range: 1 month-48.5 years). SMR was 3.6 (95\% Cl, 2.9-4.5), for those operated before 1990, 4.7 (95\% Cl, 2.7-7.6) and for those after 1990, 3.5 (95\% Cl, 2.8-4.4). Main causes of death were cardio/cerebrovascular (33.7\%), infections (30.1\%), and malignancy (28.9\%). Cox regression analysis demonstrated that only age at diagnosis remained an independent predictor of mortality (hazard ratio $1.10 ; 95 \% \mathrm{Cl}, 1.07-1.13, P<0.001$ ), whereas sex, presentation with acute apoplexy, extent of tumor removal, radiotherapy, recurrence, untreated GH deficiency, FSH/LH deficiency, ACTH deficiency, TSH deficiency, and treatment with desmopressin had no impact.

Conclusions: Despite the improvement of treatments over the last three decades, the mortality of patients with NFAs in our series remains high. Apart from age, factors related with the management/outcome of the tumor are not independent predictors, and pituitary hormone deficits managed with the currently-used substitution protocols do not adversely affect mortality.

\section{Introduction}

Non-functioning pituitary adenomas (NFAs) have a prevalence of 7-22/100 000 people $(1,2)$, a standardized incidence rate of 1.02/100 000 (3), and comprise 15-37\% of all pituitary adenomas $(1,2,3)$. They are found more commonly than previously thought, mainly due to the increased awareness of pituitary disease, as well as due to the advances and the easier access to diagnostic tools. As they are not associated with hormonal hypersecretion, they usually escape early diagnosis, and are mostly recognized when they are large enough to exert pressure effects to surrounding tissues; thus, at the time of detection, 67-90\% of them are macroadenomas with the median age of the patients ranging between 51.5 and 65.5 years $(1,2,3)$. A significant number of patients with NFAs suffer from morbidities related to the tumor, possible recurrence(s), and the treatments utilized; visual

Published by Bioscientifica Ltd. 
deterioration and hypopituitarism are the most frequent $(4,5,6)$. Studies assessing patients with NFAs following surgery and using various diagnostic criteria have shown growth hormone $(\mathrm{GH})$ deficiency in $78-88 \%$, folliclestimulating hormone (FSH)/luteinizing hormone (LH) deficiency in 46-90\%, thyroid-stimulating hormone (TSH) deficiency in 12-57\%, adrenocorticotropic hormone (ACTH) deficiency in 13-60\% (7), and persistent diabetes insipidus (DI) in 15\% (6); a further increase in these rates is expected if pituitary radiotherapy is added (8).

Studies assessing the long-term mortality of patients with NFA are very limited, and provide conflicting outcomes reporting no difference or a higher mortality compared with the general population $(9,10,11,12,13$, 14). Furthermore, the data on predictors of mortality are either conflicting (e.g. sex $(9,11,12,14)$ and radiotherapy $(11,13,14,15))$ or are not available, and importantly, the impact of hypopituitarism and its management (particularly with the currently-used hormone substitution protocols) on survival is not yet clearly defined in this group of patients with non-secreting adenomas. Drawbacks of the published reports include the relatively small number of patients studied $(10,11)$, selection bias with assessment only of subjects who received post-operative radiotherapy (13), and inclusion of other types of hypothalamic-pituitary tumors in the analysis $(9,12,13)$.

The aim of the present study was to assess the mortality and its causes in a large series of non-selected patients with macro NFAs diagnosed between January 1963 and December 2011 and followed up in a tertiary referral center in the UK, as well as parameters predicting survival with particular emphasis on possible roles of radiotherapy, recurrence, and hypopituitarism.

\section{Subjects and methods}

\section{Patients and follow-up}

The records of the patients followed-up in the Department of Endocrinology in Oxford for a non-functioning pituitary macroadenoma diagnosed between January 1963 and December 2011 were reviewed in this retrospective cohort study. These were identified from the database of the Department, in which the cases are classified according to diagnosis. The study was completely retrospective in nature and involved no intervention beyond usual patient care. It was registered with and approved as an audit by the Oxford University Hospitals Trust.
All patients had undergone pituitary surgery (transsphenoidal or by craniotomy). The extent of adenoma removal was assessed by imaging at least 3 months postoperatively and adjuvant fractionated radiotherapy was offered in cases of residual tumor based on the decision of the endocrine, neurosurgical, and oncology team. Followup imaging was performed at regular intervals and was reported by specialist neuroradiologists (since 1995, all scans were reviewed by one neuroradiologist (J V Byrne)); regrowth of the adenoma was diagnosed on the basis of radiological appearances with or without associated signs and symptoms. All the patients diagnosed before 1980 had follow-up extending during the computed tomography and magnetic resonance imaging era, thereby allowing imaging by more precise modalities. The diagnosis of apoplexy was based on the classical clinical picture combined with imaging and/or pathological findings.

Assessment of pituitary function was performed preoperatively, $6-8$ weeks post-operatively, at yearly intervals following radiotherapy (for the hormonal axes remaining intact), and when an increase in the size of the residual tumor was detected. The diagnosis of GH deficiency was based on suboptimal response of GH to dynamic testing (insulin tolerance or glucagon or GHRH + arginine testing) according to the accepted criteria at the time of assessment, or on the presence of $>2$ other pituitary hormone deficits and low serum insulin-like growth factor 1 (IGF1) levels. Gonadotropin deficiency was diagnosed on the basis of morning serum testosterone below the reference range with low or normal gonadotropins in males, low serum estradiol and oligo-/amenorrhea with low or normal gonadotropins in premenopausal females, and gonadotropins below the age related-values in postmenopausal females. ACTH deficiency was confirmed following suboptimal response of the serum cortisol to dynamic testing (insulin tolerance or glucagon or short Synacthen testing) according to the accepted criteria at the time of assessment, or after detection of an $0900 \mathrm{~h}$ serum cortisol value $<100 \mathrm{nmol} / \mathrm{l}(3.6 \mu \mathrm{g} / \mathrm{dl})$ in the absence of steroid therapy. TSH deficiency was defined by low or normal TSH with thyroid hormone levels below the reference values. DI was diagnosed on the basis of urine output $>31 / 24 \mathrm{~h}$ and urine osmolality $<300 \mathrm{mOsm} / \mathrm{kg}$ after pituitary surgery followed by a water deprivation test in cases of diagnostic uncertainty. Relevant hormonal replacement was offered to all patients with ACTH or TSH deficiency or DI; hydrocortisone was usually administered in a total daily dose of $10-25 \mathrm{mg}$ split in three doses, levothyroxine in a dose aiming to produce a free thyroxine level within the reference range (preferably in the 
upper half), and desmopressin aiming to achieve symptomatic relief and normal serum sodium levels. All males or premenopausal females with gonadotropin deficiency were treated with gonadal hormone replacement (in males with the available testosterone formulations and in females with combined estrogen/gestagen or, in cases with an absent uterus, estrogen alone), unless contraindicated or if the patient developed adverse effects on this treatment or declined it. GH deficient subjects were offered GH replacement if they fulfilled the criteria required by the UK National Institute of Clinical Excellence (16), unless contraindicated or if the patient declined this treatment. All patients underwent a gradual dose titration and the final dose used ranged between 0.2 and $1 \mathrm{mg}$ daily aiming to achieve IGF1 levels within the age- and sex-reference range (and preferably in the upper half). Subjects offered pituitary hormone replacement had at least annual biochemical assessment for confirmation of the adequacy of treatment.

\section{Mortality data}

For the assessment of mortality, status was recorded as either dead or alive, as of 31st December 2011, and data were retrieved through careful search from the National Health Service Central Register and the hospital records. Survival was estimated from the date of pituitary surgery until the date of death for those who died, or until 31st December 2011 for those who survived. The primary cause of death (1a) was established from death certificates obtained from the General Register Office in the UK, which maintains the national archive of all deaths dating back to 1837 .

\section{Statistical analyses}

Percentages were calculated for categorical data and medians with ranges for continuous variables. Standardized mortality ratios (SMRs) were estimated as the ratio of observed over expected deaths. The expected number of deaths was calculated by multiplying age and calendar year specific mortality rates for the general population of England and Wales (obtained from the Office for National Statistics) by the corresponding person-years at risk amongst the patients with NFA (17). The survival curves were generated by the Kaplan-Meier method and differences in the survival of subgroups were checked by the log-rank test. Cox regression analysis was used to assess the effect of various parameters on mortality (hazard ratios (HRs) and 95\% CIs).
The level of significance was set at $P<0.05$. Statistical analyses were performed by the SPSS 19.0 for Windows (SPSS, Inc.).

\section{Results}

\section{Overall mortality}

In total, 546 patients were identified (333 males and 213 females; median age at diagnosis 58.7 years (range: 16.1-94.2)). Transsphenoidal surgery had been offered to $532(97.4 \%)$ and craniotomy to $14(2.6 \%)$ as primary surgical treatment; adjuvant radiotherapy had been subsequently used for 159 patients (29.2\%). The median follow-up was 8 years (range: 1 month-48.5 years; eight subjects had follow-up duration of 1 month; six had surgery 1 month before the database was frozen; and two died within 1 month after the pituitary operation). The characteristics of this group are summarized in Table 1. During the follow-up period, 83 patients (52 males and 31 females) died at a median age of 77.8 years (range: 36.4-98.3). The deaths were attributed to cardio/ cerebrovascular causes $(n=28,33.7 \%)$, infections $(n=25$, $30.1 \%-24$ chest and one urinary tract; one chest infection was hospital acquired), malignancy ( $n=24,28.9 \%)$, perioperatively during pituitary surgery $(n=1,1.2 \%)$,

Table 1 Characteristics of total group of patients with NFA.

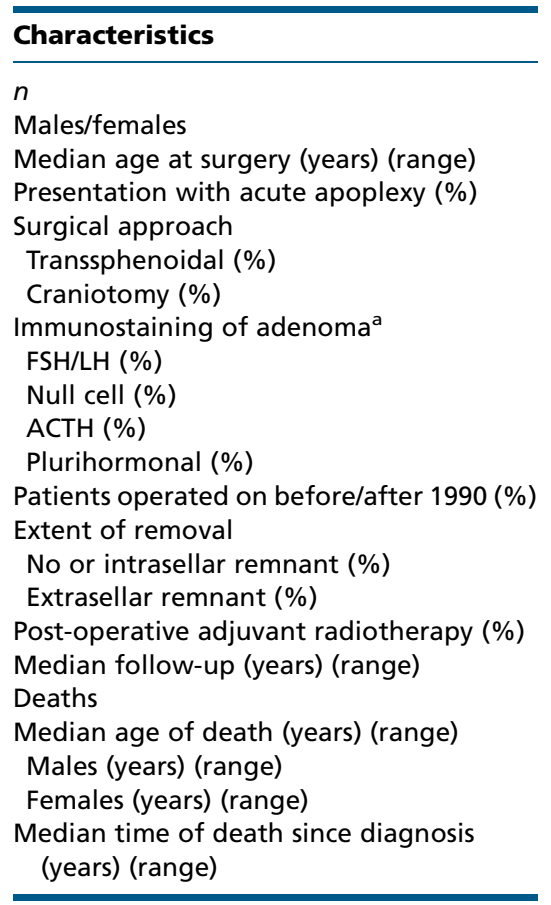

\begin{tabular}{c}
\hline Values \\
\hline 546 \\
$333 / 213$ \\
$(16.1-94.2)$ \\
$77 / 544(14.2 \%)$ \\
\\
$532 / 546(97.4 \%)$ \\
$14 / 546(2.6 \%)$ \\
\\
$276 / 487(56.7 \%)$ \\
$90 / 487(18.5 \%)$ \\
$41 / 487(8.4 \%)$ \\
$80 / 487(16.4)$ \\
$48 / 498(8.8 / 91.2 \%)$ \\
\\
$249 / 533(46.7 \%)$ \\
$284 / 533(53.3 \%)$ \\
$159 / 546(29.2 \%)$ \\
$8(1$ month-48.5 years) \\
$83 / 546(15.2 \%)$ \\
$77.8(36.4-98.3)$ \\
$78.4(51.1-92.7)$ \\
$77.7(36.4-98.3)$ \\
$7.4(1$ month-37 years) \\
\hline \\
\end{tabular}

${ }^{\text {a }}$ Available in 487 cases. 
gastrointestinal hemorrhage $(n=1,1.2 \%)$, suicide $(n=1$, $1.2 \%)$, old age $(n=1,1.2 \%)$, and 'unknown' $(n=2,2.4 \%)$ : $67 \%$ of the patients who died of infections had ACTH deficiency. The SMR for the total group of patients was 3.6 (95\% CI, 2.9-4.5); for those operated before 1990, 4.7 (95\% CI, 2.7-7.6) and for those after 1990, 3.5 (95\% CI, 2.8-4.4) (Table 2).

\section{Predictive factors of mortality}

Clinical, hormonal, and imaging follow-up data (until date of death or if alive, until date the database was frozen) were available for 436 patients (269 males and 167 females; median age at diagnosis 58.5 years (range: $16.1-$ 94.2)) (Table 3). This group was used for the estimation of SMRs related to long-term outcomes/management of the NFA and predictive factors of mortality. Transsphenoidal surgery had been offered to 423 (97\%) and craniotomy to $13(3 \%)$ as primary surgical treatment; adjuvant radiotherapy has been subsequently used for 123 patients (28.2\%). The median follow-up was 6.9 years (1 month48.5 years); during this interval, regrowth of the tumor had been detected in 110 patients (25.2\%). Detailed data on the pituitary function and the treatment of hypopituitarism are shown in Table 3.

During the follow-up period, 64 patients (52 males and 31 females) died at median age of 76.6 years (range: 36.4-90.4). The SMR estimated for this group was 3.8 (95\% CI, 2-4.7; Table 4). In patients with tumor regrowth, this was 3.5 (95\% CI, 2.1-5.5) and in those without, 3.9 (95\% CI, 2.9-5.1). SMRs for subjects treated by radiotherapy (adjuvant post-operative or for relapse) or not were 4.1 (95\% CI, 2.7-6.0) and 3.6 (95\% CI, 2.5-4.7) respectively. Those with untreated GH deficiency had SMR 3.9 (95\% CI, 2.8-5.2) and with treated GH deficiency 3.5 (95\% CI, 1.8-6.3). Patients with intact ACTH axis had SMR 3.3 (95\% CI, 2.1-4.8) and with treated ACTH deficiency 4.0 (95\% CI, 2.9-5.4). Hydrocortisone replacement showed the following dose distribution pattern: $10-<20 \mathrm{mg}$

Table 2 SMRs based on the total group of patients.

\begin{tabular}{|c|c|c|c|c|}
\hline $\begin{array}{l}\text { Groups of } \\
\text { patients }\end{array}$ & $\begin{array}{c}\text { Observed } \\
\text { deaths }\end{array}$ & $\begin{array}{c}\text { Expected } \\
\text { deaths }\end{array}$ & $\begin{array}{c}\text { SMR } \\
(95 \% \mathrm{Cl})\end{array}$ & $P$ value \\
\hline Total group & 83 & 22.9 & $3.6(2.9-4.5)$ & $<0.001$ \\
\hline $\begin{array}{l}\text { Patients } \\
\text { operated on } \\
\text { before } 1990\end{array}$ & 14 & 3 & $4.7(2.7-7.6)$ & $<0.001$ \\
\hline $\begin{array}{l}\text { Patients } \\
\text { operated on } \\
\text { after } 1990\end{array}$ & 69 & 19.6 & $3.5(2.8-4.4)$ & $<0.001$ \\
\hline
\end{tabular}

Table 3 Characteristics and long-term outcomes of patients with NFAs and adequate follow-up data (until date of death or, if alive, until date the database was frozen).

\section{Characteristics}

$n$

Males/females

Median age at surgery (years) (range)

Presentation with acute apoplexy (\%)

Surgical approach

Transsphenoidal (\%)

Craniotomy (\%)

Patients operated on before or after 1990 (\%)

Extent of removal

No of intrasellar remnant (\%)

Extrasellar remnant (\%)

Post-operative adjuvant radiotherapy ${ }^{a}(\%)$

Regrowth of tumor (\%)

Radiotherapy (adjuvant post-operative or for relapse) $)^{\mathrm{a}}(\%)$

Repeat surgery for relapse (\%)

Median follow-up (years) (range)

Deaths

Median age of death (years) (range)

$\mathrm{GH}$ status $^{\mathrm{b}}(\%)$

$\mathrm{GH}$ replete

$\mathrm{GH}$ deficient untreated

$\mathrm{GH}$ deficient treated

Unknown

FSH/LH status (\%)

$\mathrm{FSH} / \mathrm{LH}$ replete

Males

Females $^{c}$

$\mathrm{FSH} / \mathrm{LH}$ deficient untreated

Males

Females $^{d}$

FSH/LH deficient treated

Males

Females

Unknown

Males

Females

ACTH status (\%)

$\mathrm{ACTH}$ replete

ACTH deficient treated

Unknown

TSH status $(\%)^{\mathrm{b}}$

TSH replete

TSH deficient treated

Unknown

ADH status $(\%)^{\mathrm{b}}$

No DDAVP

On DDAVP

Unknown

\begin{tabular}{c}
\hline Values \\
\hline 436 \\
$269 / 167$ \\
$58.5(16.1-94.2)$ \\
$61 / 434(14.1 \%)$ \\
$423 / 436(97 \%)$ \\
$13 / 436(3 \%)$ \\
$36 / 400(8.3 / 91.7 \%)$
\end{tabular}

$202 / 431(46.9 \%)$ 229/431 (53.1\%) $123 / 436(28.2 \%)$ $110 / 436(25.2 \%)$ $188 / 436(43.1 \%)$

$41 / 436(9.4 \%)$

6.9 (1 month-48.5 years) $64 / 436(14.7 \%)$

76.6 (36.4-90.4)

$16 / 436(3.7 \%)$

$222 / 436(50.9 \%)$

$101 / 436(23.2 \%)$

$97 / 436(22.2 \%)$

$43 / 269(16 \%)$

$131 / 167(78.4 \%)$

$34 / 269(12.6 \%)$

$3 / 167(1.8 \%)$

$189 / 269(70.3 \%)$

$32 / 167(19.2 \%)$

$3 / 269(1.1 \%)$

$1 / 167(0.6 \%)$

$224 / 436(51.4 \%)$

209/436 (47.9\%)

$3 / 436(0.7 \%)$

$207 / 436(47.5 \%)$

225/436 (51.6\%)

$4 / 436(0.9 \%)$

$397 / 436(91.1 \%)$

$36 / 436(8.2 \%)$

$3 / 436(0.7 \%)$

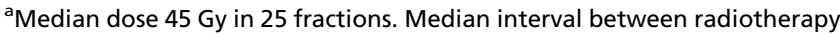
(primary or for relapse) and death or if alive, last assessment: 7.5 years (range: $0.3-48.5)$. Median age when radiotherapy (primary or for relapse): 56.7 years (range: $16.1-85.1$ ).

${ }^{\mathrm{b}}$ At last assessment.

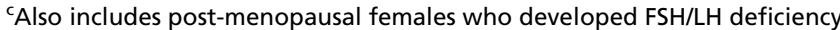
after the mean age of natural menopause.

${ }^{d}$ Also includes females who at last follow-up were aged above the age of natural menopause but had untreated FSH/LH deficiency during their reproductive life.

eOn hydrocortisone: total daily dose: $10-<20 \mathrm{mg}$ 18/209 patients, $20 \mathrm{mg}$ $170 / 209$ patients, $25-30 \mathrm{mg} \mathrm{15/209}$ patients, and unknown 6/209 patients. 
Table 4 SMRs based on the group of patients with adequate follow-up data (until date of death or if alive, until date the database was frozen).

\begin{tabular}{l} 
Groups of patients \\
\hline Total group \\
Patients with tumor regrowth \\
Patients without tumor regrowth \\
Patients with radiotherapy (adjuvant post- \\
operative or for relapse) \\
Patients without radiotherapy \\
Patients with untreated GH deficiency \\
Patients with treated GH deficiency \\
Patients with intact ACTH axis \\
Patients with treated ACTH deficiency \\
Patients with intact TSH axis \\
Patients with treated TSH deficiency \\
Patients not on desmopressin \\
Patients on desmopressin
\end{tabular}

\begin{tabular}{c}
\hline $\begin{array}{c}\text { Observed } \\
\text { deaths }\end{array}$ \\
\hline 64 \\
16 \\
48 \\
25 \\
39 \\
42 \\
10 \\
24 \\
38 \\
29 \\
33 \\
61 \\
1
\end{tabular}

\begin{tabular}{c}
$\begin{array}{c}\text { Expected } \\
\text { deaths }\end{array}$ \\
\hline 17 \\
4.6 \\
12.3 \\
6.1 \\
10.8 \\
10.9 \\
2.8 \\
7.4 \\
9.5 \\
7.2 \\
9.6 \\
16.3 \\
0.6
\end{tabular}

\begin{tabular}{l} 
P value \\
\hline 0.001 \\
$<0.001$ \\
$<0.001$ \\
$<0.001$ \\
\\
$<0.001$ \\
$<0.001$ \\
$<0.001$ \\
$<0.001$ \\
$<0.001$ \\
$<0.001$ \\
$<0.001$ \\
$<0.001$ \\
0.6
\end{tabular}

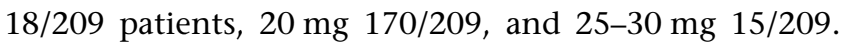
SMRs for subjects with intact TSH axis or with treated TSH deficiency were 4.2 (95\% CI, 2.7-5.7) and 3.4 (95\% CI, 2.4-4.8), respectively, and for those not on desmopressin or on desmopressin were 3.7 (95\% CI, 2.9-4.80 and 1.7 (95\% CI, 0.08-8.5) respectively (Table 4).

On univariate regression analysis, significant predictors of mortality were age at diagnosis (HR 1.10/1 year increase; 95\% CI, 1.08-1.13, $P<0.001)$, radiotherapy (adjuvant post-operative or for relapse) (HR 2.21; 95\% CI, 1.31-3.73, $P<0.001$ ), regrowth of the tumor (HR 1.99; $95 \% \mathrm{CI}, 1.11-3.56, P=0.02)$, and untreated $\mathrm{GH}$ deficiency (HR 0.32; 95\% CI, 0.16-0.64, P=0.01), whereas sex, presentation with acute apoplexy, cavernous sinus invasion, extent of adenoma removal, repeat surgery for relapse, untreated FSH/LH deficiency, ACTH deficiency, TSH deficiency, and treatment with desmopressin were not. Multivariate analysis including the variables found to be significant showed that only age at diagnosis remained an independent predictor of mortality (HR 1.10/1 year increase; 95\% CI, 1.07-1.13, $P<0.001)$. There was a significant difference in the rate of survival between those diagnosed before the age of 50 years and after the age of 50 (at 10 years follow-up $98.5 \%$ vs $80.9 \%$ respectively $P<0.01$; Fig. 1 ).

\section{Discussion}

This is the first study assessing the SMR of patients with NFAs based on the largest series of non-selected subjects followed-up in a single UK tertiary referral center during a long period (median 8 years). All our subjects had histopathologically confirmed diagnosis and underwent a very detailed, comprehensive clinical characterization, particularly on the tumor treatment, the degree of hypopituitarism and its management. We found that mortality was 3.6 times higher than that of the general population and that the main causes of death were cardio/cerebrovascular, infections, and malignancies. Despite the improvement in mortality in the group of patients operated after 1990, the SMRs remained high; of the number of factors assessed, only age at diagnosis was an independent significant predictor and, importantly, pituitary hormone deficits managed with mostly current protocols did not affect mortality.

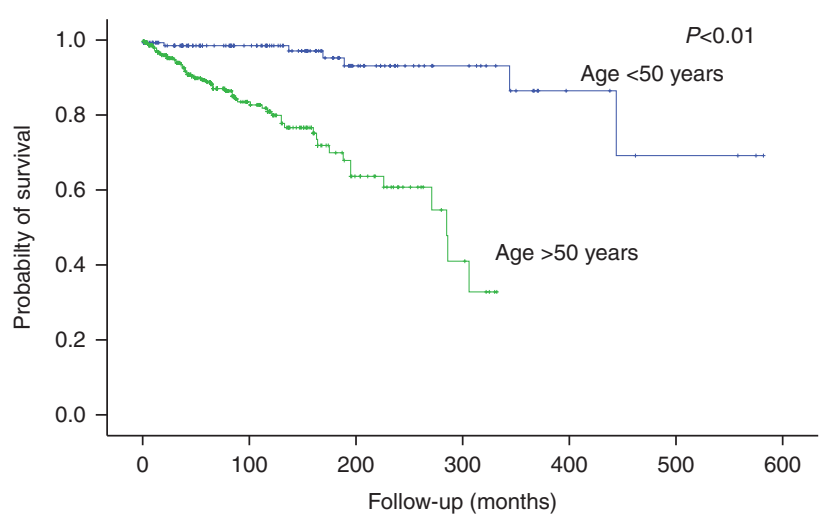

\section{Figure 1}

Cumulative probability of survival between those diagnosed before and after the age of 50 years; a significant difference was found (log-rank test; at 10 -year follow-up $98.5 \%$ vs $80.9 \%$ respectively $P<0.01$ ). A full colour version of this figure is available at http://dx.doi.org/10.1530/EJE-15-0967. 
Studies assessing the long-term mortality of patients with NFAs are scarce and provide conflicting data. Lindholm et al. (10), in a series of 160 patients with NFAs operated between 1985 and 1996 (18\% had adjuvant radiotherapy) and followed-up for a median period of 12.4 years, found an SMR of 1.18 (95\% CI, 0.87-1.60). Similar results were reported by Nielsen et al. (11) in 192 patients treated between 1985 and 1996 (22\% received adjuvant radiotherapy; SMR 1.21 (95\% CI, 0.93-1.59) - median follow-up for the surviving subjects 13.1 years and for those who died 4.6 years). The relatively small number of patients remains a limitation of both reports. Bates et al. (9), in a group of 348 patients with pituitary tumors, most of which were NFAs, treated between 1970 and 1992 (28\% had adjuvant radiotherapy), found an SMR of 1.2 (95\% CI, 0.95-1.55). Notably, a reduction in cardiovascular mortality was identified; a rather surprising finding given that $94 \%$ of the subjects had ACTH deficiency and 91\% of them were treated with hydrocortisone doses of $30 \mathrm{mg} /$ day, which would be considered overtreatment according to modern management criteria. On the other hand, Chang et al. (14), in a series of 663 patients with NFAs managed between 1975 and 1995 (51\% received adjuvant radiotherapy) and followed up for a median period of 14 years, reported that the study population had a significantly higher mortality rate than the general population; however, no SMRs were provided. Finally, Brada et al. (13) in a group of 334 patients with pituitary adenomas, two-thirds of which were NFAs treated with radiotherapy (following surgery or as primary approach, usual dose 40-50 Gy in 20-30 fractions with conventional three-field techniques between 1962 and 1986), found a relative risk of death of 1.58 (95\% CI, 1.32-1.90) compared with the general population. Notably, the relative risk of cerebrovascular deaths in patients with NFAs was 3.65 (95\% CI, 2.26-5.58). This study suffers from selection bias, as it included only patients referred for radiotherapy and had no details on pituitary function and treatment of possible hormone deficits. It should be noted that the high SMR we identified may also relate to the extensive search of deaths we performed (through both National Health Service Central Register and the hospital records) which allowed us capture all deceased patients.

In our study, the SMR of the patients operated after 1990 was found 3.5, whereas for those treated before this period it was 4.7. This improvement is in agreement with Nilsson et al. (12) and the meta-analysis by Nielsen et al. (18), and possibly relates to the advances in the diagnosis and management of pituitary disease including hormonal replacement. Amongst a number of factors assessed and following multivariate analysis, only age at diagnosis remained an independent predictor of mortality (shorter survival for older patients) and the probability of survival was significantly lower in subjects aged more than 50 years. Nilsson et al. (12) and Chang et al. (14) have also confirmed the negative impact of age at presentation. Furthermore, acute apoplexy and extent of resection have not been found as independent predictors in previous series $(14,19)$.

The causal link between pituitary irradiation and mortality (particularly cerebrovascular) remains a subject of debate (20) and relevant randomized trials are not available. In our study, the median follow-up after radiotherapy was 7.5 years (range: 0.3-48.5), and this treatment modality did not affect survival. Although, Brada et al. (13) reported high SMRs in patients with various types of irradiated pituitary adenoma, this study lacked a control group. On the other hand, series including only patients with NFAs are in agreement with our results. Thus, Nielsen et al. (11) reported that the SMR of patients with NFAs who had received radiotherapy was similar to that of those treated solely by surgery. Similarly, Chang et al. (14) showed that radiotherapy was not an independent predictor of survival, while Tomlinson et al. (15) in a series of 573 patients with NFAs included in the West Midlands Hypopituitary database found no effect of radiotherapy on mortality.

In our series, based on a non-selected group of patients which, importantly, did not include any type of functioning adenomas, we found that hypopituitarism is not an independent predictor of mortality. The advances in the management of pituitary hormone deficits (earlier diagnosis, more optimal hormone substitution, and closer monitoring) may have contributed to this. Previously published observational studies on this topic have a number of drawbacks, making the interpretation of their results rather difficult; thus, they rely on series of small number of patients $(10,21)$, with occasionally rather outdated diagnostic criteria $(21,22,23)$ and different or suboptimal hormonal regimes (often higher doses of steroids than the ones currently used, non-routine sex hormone substitution or GH) $(9,15,21,22,23,24)$. These factors may influence the reported outcomes and the conclusions of studies drawn mainly from early calendar periods do not necessarily reflect the prognosis of patients managed in the latest years $(9,21,22,23)$. Furthermore, a number of series include heterogeneous groups of underlying causes of hypopituitarism (e.g. functioning adenomas and craniopharyngiomas) managed with various modalities having the potential to affect mortality 
independently of pituitary hormone deficits $(9,21,22,23$, 24, 25). Tomlinson et al. (15) in a series of 573 patients with NFAs included in the West Midlands Hypopituitary database found an SMR of 1.70 (99\% CI, 1.34-2.15) with the excess mortality explained by an increase in respiratory and vascular deaths. Patients with untreated gonadotropin deficiency had a worse outcome compared with those with treated deficiency; notably, the percentage of subjects with untreated gonadotropin deficiency was $37 \%$ compared with only $14 \%$ in our study. It should be also pointed out that selection bias may be present in this series, as it does not include consecutive NFAs but only those with hypopituitarism.

In our study, ACTH deficiency was not a predictor of mortality; notably, in around $90 \%$ of our patients the total daily dose of hydrocortisone was $20 \mathrm{mg}$. This is in agreement with Zueger et al. (26) who, in a series of 105 patients with NFAs and ACTH deficiency, found that the HR for mortality was one for those treated with a total dose between 5 and $19 \mathrm{mg}$ daily but increased to 2.03 for total dose in the range of 20-29 mg. Furthermore, based on data of hypopituitary patients from the KIMS cohort (Pfizer International Metabolic Database) (27), ACTH deficient patients on hydrocortisone-equivalent doses of $<20 \mathrm{mg} /$ day do not differ in their metabolic profile from the ACTH deplete. Given our small number of patients on total daily dose $>20 \mathrm{mg}$, we did not analyze the impact of total dose on mortality. Interestingly, $67 \%$ of our patients who died of infections had ACTH deficiency, and a contribution of adrenal insufficiency cannot be excluded.

Multivariate analysis did not confirm GH status as an independent predictor of mortality. It has been shown that $\mathrm{GH}$ replacement improves cardiovascular risk factors but to date, only a limited number of studies (mainly postmarketing surveillance) have investigated its effect on mortality in GH deficient adults and long-term controlled trials on mortality (including cardiovascular) are lacking (28). Svensson et al. (29) in a series of 1411 adult hypopituitary patients not on $\mathrm{GH}$ replacement found mortality higher than that of the normal population (risk ratio 3.36; 95\% CI, 2.93-3.83) with increased rates of myocardial infarctions, cerebrovascular events, and malignancies. In the same report, patients on GH replacement had overall mortality similar to the normal population. On the other hand, data from the Dutch National Registry of Growth Hormone Treatment in Adults have suggested that mortality in patients treated with $\mathrm{GH}$ was high in females mainly due to cardiovascular disease, whereas, in the control group of untreated subjects this was not different from the background population (30). In both studies however, information on the underlying causes of hypopituitarism and its management, as well as details on the presence of other pituitary hormone deficits and their treatment are not included. It should be noted that, in our center, GH-deficient patients are offered GH replacement after assessment of their quality of life with a standardized questionnaire (Assessment of Growth Hormone Deficiency in Adults (AGHDA)). Those with optimal scores are not candidates for treatment and, therefore, there is necessarily a selection bias introduced by the prescreening of candidates for $\mathrm{GH}$ replacement according to the NICE guidelines; this needs to be taken into account when considering the overall impact of GH replacement on mortality in this series.

Very recently, while this manuscript was being prepared for submission, a study of 2795 patients with NFA followed in nationwide health registries in Sweden between 1987 and 2011 was published reporting SMR of 1.10 (95\% CI, 1.00-1.20) (31). The validity of the diagnosis is a drawback of this report as the identification criteria checked in a sample of 467 subjects confirmed NFA in 424 (90.8\%) and importantly, 1172 patients diagnosed between 1997 and 2011 had received no surgical treatment or radiotherapy, thereby lacking histological confirmation (notably, information on surgical treatments and radiotherapy were not available for those diagnosed before 1997). Furthermore, the SMR for patients with hypopituitarism (defined by entry of this term in the patient registry) was found 1.06 (95\% CI, 0.94-1.19); nonetheless, data on the diagnosis and degree of hypopituitarism, the percentage of subjects tested, as well as its management factors of major importance when assessing outcome - are not available. Finally, in contrast to our detailed clinical characterization which allowed assessment of the impact of factors like acute apoplexy, extent of NFA removal and regrowth, the recently published series evaluates the effects of the total number of treatments offered for the NFA without providing or taking into account details on the natural history of each tumor and the reason the treatments were used (for primary adenoma or for relapse).

The large sample size of non-selected patients with histologically confirmed NFAs followed up for a long period in one center and managed with similar (and mostly current) protocols in terms of hormone substitution are the strengths of our study, whereas limitations include its retrospective nature, the selection bias in GH treated patients, and the unknown duration of pituitary hormone deficits prior to their diagnosis (which is however, practically not possible to be clarified). 
In conclusion, patients operated for an NFA have increased mortality and factors related with the management/outcome of the tumor including presentation with acute apoplexy, extent of adenoma removal, radiotherapy, and recurrence do not seem to be independent predictors. We have also shown that pituitary hormone deficits managed with the currently used substitution protocols do not adversely affect mortality, although the impact of $\mathrm{GH}$ replacement on survival remains to be assessed with more robust studies specifically designed to address this question. The only parameter affecting mortality in the patients followed-up in our center was age at diagnosis, a finding also supported by previous studies. The pathophysiological mechanisms of this or alternative explanations for the increased mortality remain to be elucidated. However, it is reassuring that current treatment modalities and replacement therapies for these tumors do not adversely affect survival.

\section{Declaration of interest}

The authors declare that there is no conflict of interest that could be perceived as prejudicing the impartiality of the research reported.

\section{Funding}

Dr G Ntali has been rewarded a Clinical Department Visit Grant from the Society for Endocrinology (UK).

\section{Acknowledgements}

We are grateful to the critical reading by Prof. Wiebke Arlt.

\section{References}

1 Daly AF, Rixhon M, Adam C, Dempergioti A, Tichomirowa MA \& Beckers A. High prevalence of pituitary adenomas: a cross-sectional study in the province of Liege, Belgium. Journal of Clinical Endocrinology and Metabolism 200691 4769-4775. (doi:10.1210/jc.2006-1668)

2 Fernandez A, Karavitaki N \& Wass JA. Prevalence of pituitary adenomas: a community-based, cross-sectional study in Banbury (Oxfordshire, UK). Clinical Endocrinology 201072 377-382. (doi:10.1111/j.1365-2265.2009. 03667.x)

3 Raappana A, Koivukangas J, Ebeling T \& Pirila T. Incidence of pituitary adenomas in Northern Finland in 1992-2007. Journal of Clinical Endocrinology and Metabolism 201095 2468-2475. (doi:10.1210/ jc.2010-0537)

4 Nomikos P, Ladar C, Fahlbusch R \& Buchfelder M. Impact of primary surgery on pituitary function in patients with non-functioning pituitary adenomas - a study of 721 patients. Acta Neurochirurgica 2004 146 27-35. (doi:10.1007/s00701-003-0174-3)

5 Dekkers OM, Pereira AM, Roelfsema F, Voormolen JH, Neelis KJ, Schroijen MA, Smit JW \& Romijn JA. Observation alone after transsphenoidal surgery for non-functioning pituitary macroadenoma.
Journal of Clinical Endocrinology and Metabolism 200691 1796-1801. (doi:10.1210/jc.2005-2552)

6 O'Sullivan EP, Woods C, Glynn N, Behan LA, Crowley R, O'Kelly P, Smith D, Thompson CJ \& Agha A. The natural history of surgically treated but radiotherapy-naive nonfunctioning pituitary adenomas. Clinical Endocrinology 200971 709-714. (doi:10.1111/j.1365-2265. 2009.03583.x)

7 Dekkers OM, Pereira AM \& Romijn JA. Treatment and follow-up of clinically nonfunctioning pituitary macroadenomas. Journal of Clinical Endocrinology and Metabolism 200893 3717-3726. (doi:10.1210/ jc.2008-0643)

8 Ntali G \& Karavitaki N. Efficacy and complications of pituitary irradiation. Endocrinology and Metabolism Clinics of North America 2015 44 117-126. (doi:10.1016/j.ecl.2014.10.009)

9 Bates AS, Bullivant B, Sheppard MC \& Stewart PM. Life expectancy following surgery for pituitary tumours. Clinical Endocrinology 199950 315-319. (doi:10.1046/j.1365-2265.1999.00650.x)

10 Lindholm J, Nielsen EH, Bjerre P, Christiansen JS, Hagen C, Juul S, Jørgensen J, Kruse A, Laurberg P \& Stochholm K. Hypopituitarism and mortality in pituitary adenoma. Clinical Endocrinology 200665 51-58. (doi:10.1111/j.1365-2265.2006.02545.x)

11 Nielsen EH, Lindholm J, Laurberg P, Bjerre P, Christiansen JS, Hagen C, Juul S, Jørgensen J, Kruse A \& Stochholm K. Nonfunctioning pituitary adenoma: incidence, causes of death and quality of life in relation to pituitary function. Pituitary 200710 67-73. (doi:10.1007/s11102-0070018-x)

12 Nilsson B, Gustavasson-Kadaka E, Bengtsson BA \& Jonsson B. Pituitary adenomas in Sweden between 1958 and 1991: incidence, survival, and mortality. Journal of Clinical Endocrinology and Metabolism $2000 \mathbf{8 5}$ 1420-1425.

13 Brada M, Ashley S, Ford D, Traish D, Burchell L \& Rajan B. Cerebrovascualr mortality in patients with pituitary adenoma. Clinical Endocrinology 200257 713-717. (doi:10.1046/j.1365-2265. 2002.01570.x)

14 Chang EF, Zada G, Kim S, Lamborn KR, Quinones-Hinojosa A, Tyrrell JB, Wilson CB \& Kunwar S. Long-term recurrence and mortality after surgery and adjuvant radiotherapy for non-functional pituitary adenomas. Journal of Neurosurgery 2008 108 736-745. (doi:10.3171/JNS/ 2008/108/4/0736)

15 Tomlinson JW, Holden N, Hills RK, Wheatley K, Clayton RN, Bates AS, Sheppard MC \& Stewart PM. Association between premature mortality and hypopituitarism. West Midlands Prospective Hypopituitary Study Group. Lancet 2001357 425-431. (doi:10.1016/S0140-6736 (00)04006-X)

16 National Institute for Clinical Excellence UK. Human growth hormone (somatropin) in adults with growth hormone deficiency. NICE technology appraisal guidance 64, 2003.

17 Breslow NE \& Day NE. In Statistical Methods in Cancer Research. Volume II. The Design and Analysis of Cohort Studies, pp 1-406. IARC Scientific Publications, 1987.

18 Nielsen EH, Lindholm J \& Laurberg P. Excess mortality in women with pituitary disease: a meta-analysis. Clinical Endocrinology 200767 693-697. (doi:10.1111/j.1365-2265.2007.02947.x)

19 Nielsen EH, Lindholm J, Bjerre P, Christiansen JS, Hagen C, Juul S, Jørgensen J, Kruse A \& Laurberg P. Frequent occurrence of pituitary apoplexy in patients with non-functioning pituitary adenoma. Clinical Endocrinology 200664 319-322. (doi:10.1111/j.1365-2265. 2006.02463.x)

20 Ayuk J \& Stewart PM. Mortality following pituitary radiotherapy. Pituitary 2009 12 35-39. (doi:10.1007/s11102-007-0083-1)

21 Bates AS, Van't Hoff W, Jones PJ \& Clayton RN. The effect of hypopituitarism on life expectancy. Journal of Clinical Endocrinology and Metabolism 199681 1169-1172.

22 Rosen T \& Bengtsson BA. Premature mortality due to cardiovascular disease in hypopituitarism. Lancet 1990336 285-288. (doi:10.1016/ 0140-6736(90)91812-O) 
23 Bulow B, Hagmar L, Mikoczy Z, Nordstrom CH \& Erfurth EM. Increased cardiovascular mortality in patients with hypopituitarism. Clinical Endocrinology 199746 75-81. (doi:10.1046/j.1365-2265.1997.d011749.x)

24 Fernandez-Rodriguez E, Lopez-Raton M, Andujar P, Martinez-Silva IM, Cadarso-Suarez C, Casanueva F \& Bernabeu I. Epidemiology, mortality rate and survival in a homogeneous population of hypopituitary patients. Clinical Endocrinology 201378 278-284. (doi:10.1111/j.13652265.2012.04516.x)

25 Burman P, Mattsson AF, Johannsson G, Höybye C, Holmer H, Dahlqvist P, Berinder K, Engström BE, Ekman B, Erfurth EM et al. Deaths among adult patients with hypopituitarism: hypocortisolism during acute stress, and de novo malignant brain tumors contribute to an increased mortality. Journal of Clinical Endocrinology and Metabolism 201398 1466-1475. (doi:10.1210/jc.2012-4059)

26 Zueger T, Kirchner P, Herren C, Fischli S, Zwahlen M, Christ E \& Stettler C. Glucocorticoid replacement and mortality in patients with nonfunctioning pituitary adenoma. Journal of Clinical Endocrinology and Metabolism 201297 E1938-E1942. (doi:10.1210/jc.2012-2432)

27 Filipsson H, Monson JP, Koltowska-Häggström M, Mattsson A \& Johannsson G. The impact of glucocorticoid replacement regimens on metabolic outcome and comorbidity in hypopituitary patients.
Journal of Clinical Endocrinology and Metabolism 200691 3954-3961. (doi:10.1210/jc.2006-0524)

28 Gazzaruso C, Gola M, Karamouzis I, Giubbini R \& Guistina A. Cardiovascular risk in patients with growth hormone (GH) deficiency and following substitution with GH - an update. Journal of Clinical Endocrinology and Metabolism 201499 18-29. (doi:10.1210/jc.20132394)

29 Svensson J, Bengtsson BA, Rosen T, Oden A \& Johannsson G. Malignant disease and cardiovascular morbidity in hypopituitary adults with or without growth hormone replacement therapy. Journal of Clinical Endocrinology and Metabolism 200489 3306-3312. (doi:10.1210/ jc.2003-031601)

30 van Bunderen C, van Nieuwpoort IC, Arwert LI, Heymans MW, Franken AM, Koppeschaar HP, van der Lely AJ \& Drent ML. Does growth hormone replacement therapy reduce mortality in adults with growth hormone deficiency? Data from the Dutch National Registry of Growth Hormone Treatment in Adults Journal of Clinical Endocrinology and Metabolism 201196 3151-3159. (doi:10.1210/jc.2011-1215)

31 Olsson DS, Nilsson AG, Bryngelsson IL, Trimpou P, Johannson G \& Andersson E. Excess mortality in women and young adults with nonfunctioning pituitary adenoma - a Swedish nationwide study. Journal of Clinical Endocrinology and Metabolism 2015100 2651-2658. (doi:10. 1210/jc.2015-1475)

Received 29 September 2015

Revised version received 4 November 2015

Accepted 6 November 2015 